\title{
COMMUNICATION
}

\section{Eliashberg Awarded 2016 Crafoord Prize in Mathematics}

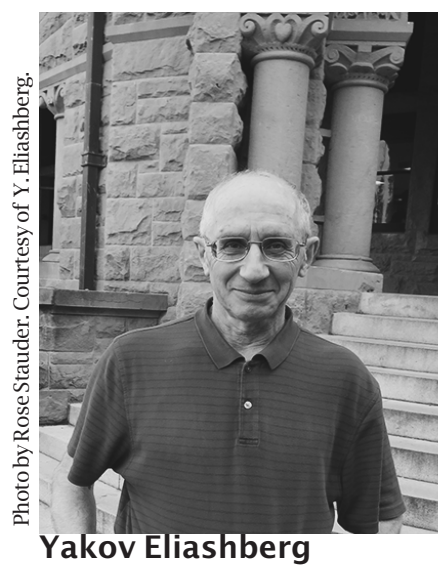

The 2016 Crafoord Prize in Mathematics has been awarded to YAKOV ELIASHBERG of Stanford University "for the development of contact and symplectic topology and groundbreaking discoveries of rigidity and flexibility phenomena".

\section{Citation}

Yakov Eliashberg has solved many of the most important problems in the field and found new and surprising results. He has further developed the techniques he used in contact geometry, a twin theory to symplectic geometry. While symplectic geometry deals with spaces with two, four, or other even dimensions, contact theory describes spaces with odd dimensions. Both theories are closely related to current developments in modern physics, such as string theory and quantum field theory.

Symplectic geometry's link to physics has old roots. For example, it describes the geometry of a space in a mechanical system, the space phase. For a moving object, its trajectory is determined each moment by its position and velocity. Together, they determine a surface element that is the basic structure of symplectic geometry. The geometry describes the directions in which the system can develop; it describes movement. Physics becomes geometry. One of Yakov Eliashberg's first and perhaps most surprising results was the discovery

For permission to reprint this article, please contact: reprint-permission@ams.org.

DOI: http://dx.doi.org/10.1090/noti1369 that there are regions where symplectic geometry is rigid and other regions where it is completely flexible. But where the boundary is between the flexible and the rigid regions, and how it can be described mathematically, is still a question that is awaiting an answer.

\section{Biographical Sketch}

Yakov Eliashberg is the Herald L. and Caroline L. Ritch Professor of Mathematics at Stanford University. Born in Russia in 1946, he received his $\mathrm{PhD}$ from Leningrad University in 1972 under the direction of Vladimir Rokhlin. He moved to the United States in 1988 and has been at Stanford since 1989. He received the Leningrad Mathematical Prize in 1972 and a Guggenheim Fellowship in 1995. He has been an invited speaker at the International Congress of Mathematicians in 1986 and 1998 and has been the recipient of a number of lectureships. He was awarded the AMS Veblen Prize in Geometry in 2001 and the Heinz Hopf Prize in 2013. Eliashberg was elected to the US National Academy of Sciences in 2003 and became an AMS Fellow in 2012.

\section{About the Prize}

The Crafoord Prize in Mathematics is awarded by the Royal Swedish Academy of Sciences approximately every three years. It is intended to promote international basic research in several disciplines of science, including astronomy, geosciences, biosciences (particulary ecology), and polyarthritis, as well as mathematics. These disciplines were chosen to complement those for which Nobel Prizes are awarded. The prize carries a cash award of 6 million Swedish krona (approximately US $\$ 700,000)$. The prize is awarded at a ceremony in Stockholm. 